\title{
Detection and scaling of statistical differences between visual textures'
}

\author{
PETER H. STOLOFF ${ }^{2}$ \\ CENTER FOR NAVAL ANALYSES: \\ AN AFFILIATE OF THE UNIVERSITY OF ROCHESTER
}

Stochastically textured patterns were used to investigate the sensitivity of $\boldsymbol{S s}$ to differences in the statistical distributions of locally defined properties of element density and shape. The results of the study indicate that when the textures were most structured, in terms of their variance, Ss were most accurate at detecting dissimilarities between pairs of patterns. Ss also rated the similarity of the statistical distributions of elements of patterns differing in local properties. Multidimensional scaling analysis of the ratings showed two dimensions, representing monotonic orderings of the stimuli.

A number of investigators (Gibson, 1950; Pickett, 1967; Rosenfeld, 1967) have conducted studies concerning the visual processes involved in texture perception. Pickett (1968) has summarized the research in a literature survey. The present research was concerned with the perception of visual textures generated and printed by computer techniques. A pattern is said to be textured when it is composed of a large number of simple patterns. The extent to which the simple patterns differ from one another and the manner in which they are spaced within the overall pattern determines the textured quality of the pattern. The process by which the simple patterns repeat thernselves may be either a deterministic or a stochastic one. Deterministic textures are categorized by the pattern generator repeating the same cycle or simple pattern with a fixed spacing over an interval. Stochastic textures are created by randomly sampling a series of simple patterns from a population containing similar patterns and/or using a random procedure for determining the spacing between cycles.

In this study, textured patterns were stochastically generated by controlling the statistics of a given, locally defined property of the simple patterns. Of specific interest is the problem of whether or not there are comparable scales of subjective statistical estimation for different local properties.

Two local properties were used in this study: number of dots and shape. In the first case, the simple patterns were clusters of dots; in the second case, they were shapes formed by two perpendicular line segments consisting of closely spaced dots. A display consisted of a pair of textured patterns, each of which was a 10 by 10 matrix of simple patterns. Each pattern in a display had approximately the same number of dots, thus keeping mean dot number, or density, constant across displays. The density values chosen for the experiments were $3,6,9,12$, and 15 dots when dot density was the local property. Associated with. a simple pattern of a given number of dots was a probability of its appearing in the textured pattern. We restricted the probabilities as follows: We let all of the density values have equal probability
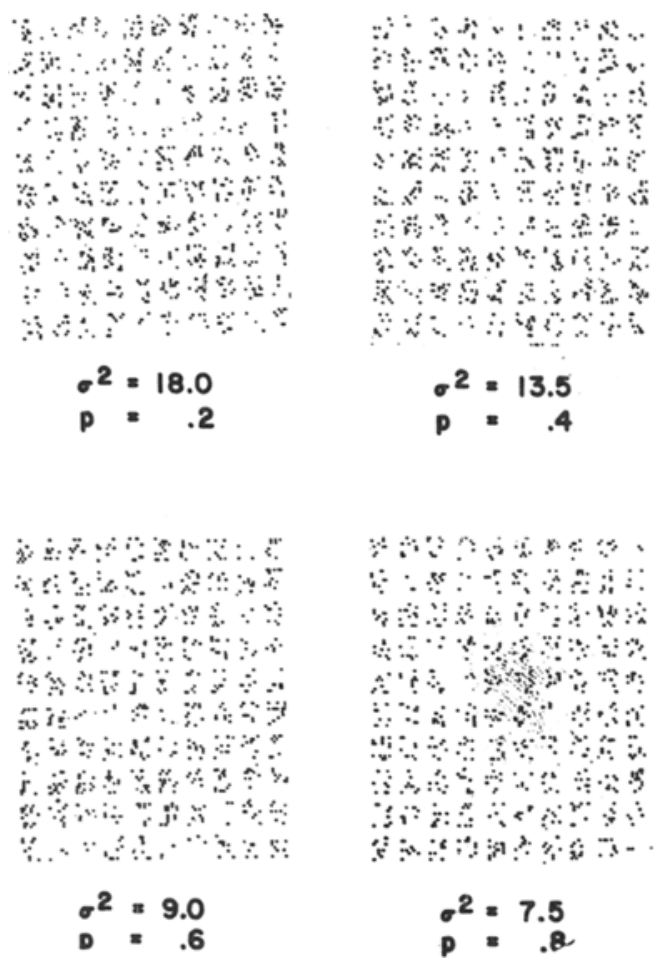

Fig. 1. Sample dot density patterns.
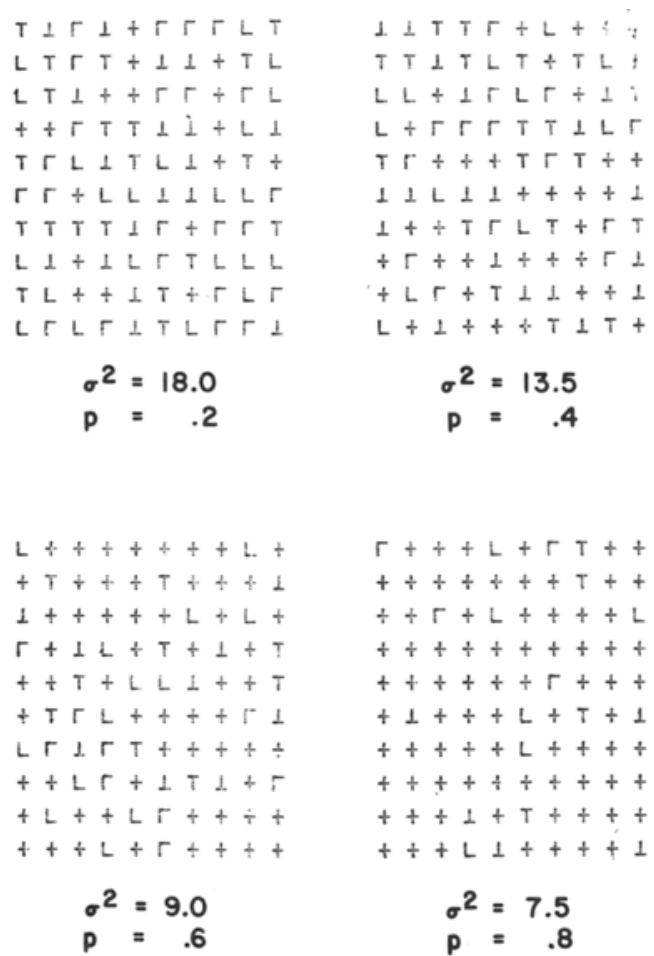

Fig. 2. Sample "shapes" patterns. 


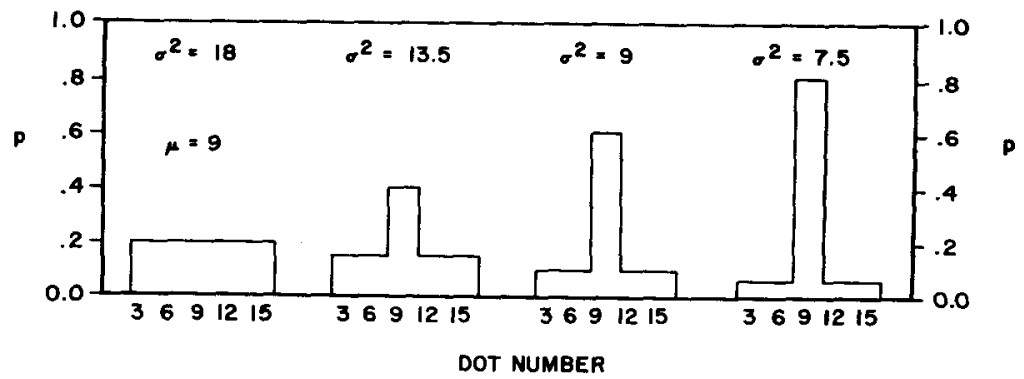

Fig. 3. Histogram of distributions of dot patterns.

of occurrence, except for the density value selected as the mean, which was 9 dots. The probability of the mean density value appearing in the textured pattern was always greater than or equal to the other densities. Changes in the value of the probability of the mean density pattern will result in changes in the even-ordered moments of the distribution of densities. Four different values for the probability of the mean density were selected, $(\mathrm{Pm}=) .20, .40, .60$, and .80 . As the $P$ value assigned to the mean density pattern increases, the variance will decrease. For example, see Fig. 3.

Patterns having shapes as a local property were constructed in a similar manner. Probability values were associated with the occurrence of each of five different shapes. The overall mean density for the shape displays was equal to that of the dot density displays so that the comparison of patterns across local properties could be made' with reference to similar statistical distributions.

Two series of shape displays were constructed, in each of which a different shape was chosen to be the most frequently occurring. In one series of patterns, a greater proportion of the +like figure occurred; in the second series, a greater proportion of the T-like figure occurred. The $P$ values were the same as in the case of the randomly placed dot patterns. The "structuredness" of a textured pattern was determined by the variance of the distribution of values for the local property. The visual angle subtended by the displays, and the duration of presentation of the display, were manipulated as independent variables, as it was felt that these variables would have a differential effect on the perception of the statistics of the textured patterns for the two local properties.

The experiments consisted of two parts: a detection study, and a scaling study.

\section{DETECTION STUDY}

Five Ss participated in the detection study. The task was to detect similarities and differences between pairs of simultaneously presented textured patterns. A set of 18 stimulus pairs, 6 having dissimilar statistical distributions, 4 having similar statistical distributions, and 8 having similar configurations of dots or shapes in corresponding cells of the matrices for these pairs of patterns were used for each local property. These were tachistoscopically presented for $100 \mathrm{msec}$, $1 \mathrm{sec}$, and using a self-paced mode. Two visual angles for the patterns were chosen, one that was close to the limits of acuity, which should allow Ss to fuse the elements in the random dot density patterns, but large enough for the shapes to be readily discernable. The larger visual angle was chosen so that the elements of the random dot density patterns were easily resolved. Per cent of correct detections and latency of response were used as dependent measures.

The five female undergraduates who were used as Ss each repeated the detection of all stimulus pairs six times. An inspection of the data, however, revealed that the first two trial periods had a high degree of variability for most Ss. These practice periods, therefore, were not included in the final detection analyses. Thus, four replications, for five $\mathrm{Ss}$, constituted the basic detection data.

\section{Results}

The results of the detection study indicated that when the patterns were most structured, Ss were most accurate at the detection task, whether they had to detect similarities or dissimilarities between patterns. Ss took less time to respond to the detection task for the highly structured patterns than for the random ones. However, a large amount of variability was evidenced in the response latency data.

The effects of manipulating the local property, visual angle, and duration of presentation independent variables were briefly:

(1) Ss were more accurate detectors of similarities than of differences in the variance of the distribution of pairs of textured displays.

(2) Ss were more accurate at the detection task when shape, rather than dot density, was the local property being manipulated.

(3) Although Ss were no more accurate at the detection task when the visual angle was manipulated, the results for the two local properties were more similar at the smaller visual angle than at the larger visual angle.

(4) Ss took a longer time to respond if given more time to view the displays, but they were not necessarily more accurate in their responses. Thus, response latency seems to reflect a measure of observer strategy rather than anything specific to the task itself.

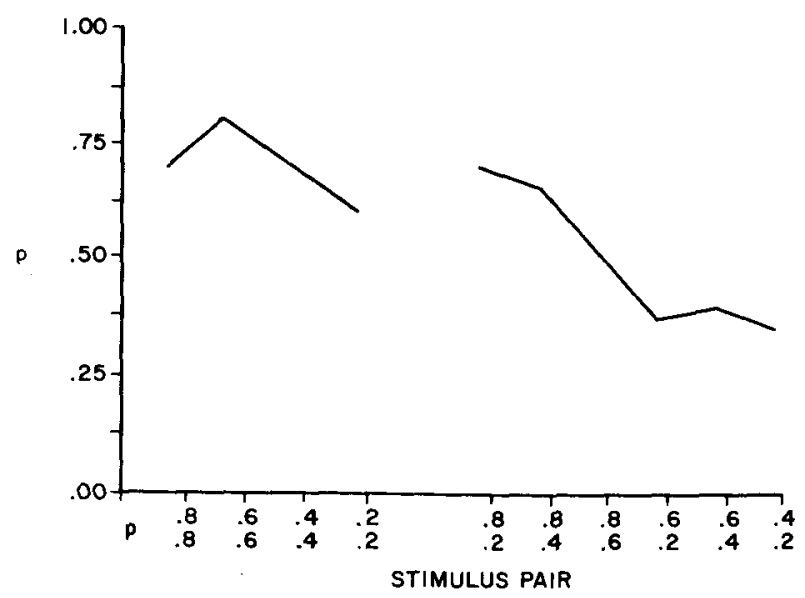

Fig. 4. Proportion of correct detections: combined data. 


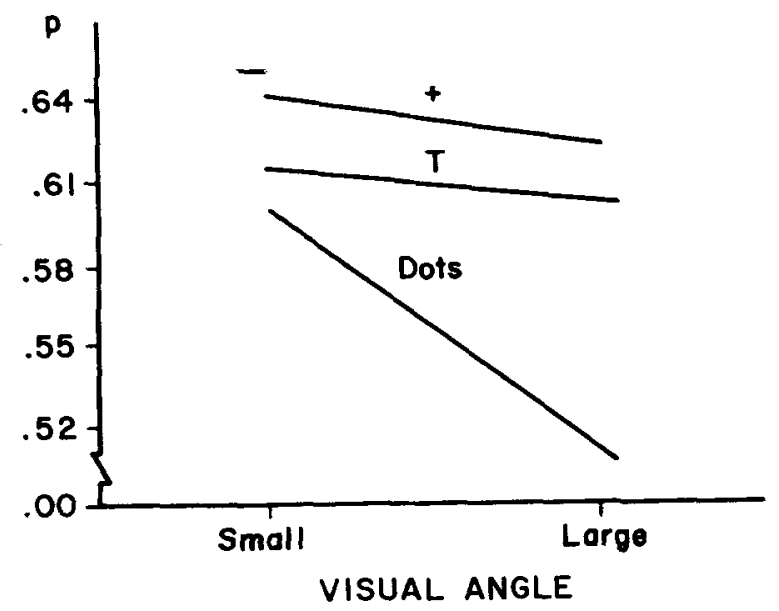

Fig. 5. Simple effects for local properties presented at different visual angles: proportion correct data.

\section{SCALING STUDY}

The second part of the study was an investigation of how Ss scale differences between textured displays where local properties are different but where the response asked for is made to differences in the statistical distributions of the local properties.

The stimuli used in the scaling study were the 28 possible pairs of a set comprised of four random dot density patterns, and four patterns containing figures with the +like figure as the most frequently occurring shape. The distributions of elements in these patterns were the same as for those patterns used for the detection study, and the distributions used for the random dot density patterns were the same as those used for the shape patterns. The patterns were presented at the larger visual angle, so as to make the local properties as perceptually different as possible as the detection data would suggest. The Ss viewed the patterns for $1.5 \mathrm{sec}$, which was about the average time it took the Ss to respond during the detection task when self-paced.

A second group of 17 Ss was chosen for the scaling study. In addition, those Ss who participated in the detection study were

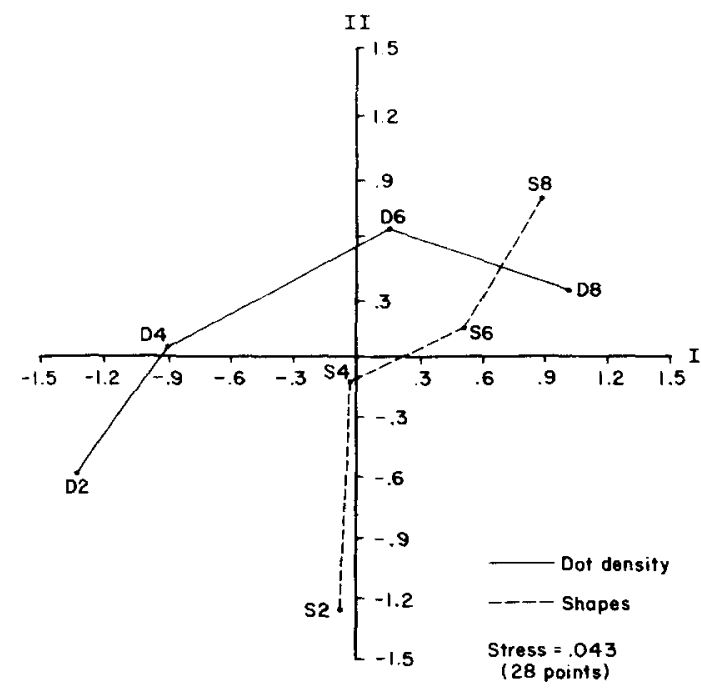

Fig. 6. Nonmetric dimensions; no-practice group. also asked to scale the stimuli. Ss were instructed to rate the similarity of the statistical distributions of elements of each pair of patterns on a 1-7 scale, where 7 was "most similar" and 1 was "least similar." The Ss who participated in the detection study repeated the scaling study a total of five times.

\section{Results}

Two multidimensional scaling methods were used to analyze the data: the so-called "traditional method" as described by Torgerson (1958), and the "nonmetric method" described by Shepard (1962) and by Kruskal (1964). Relative interstimulus distances were determined by using the procedure described by Diederich, Messick, and Tucker (1957). These same distances were then converted to $P$ values, and were used as measures of experimental similarities for the nonmetric analysis by the computer programs provided by J. B. Kruskal.

The response data from the practiced and the new group of Ss were analyzed separately. The nonmetric solution for the unpracticed group of $S s$ resulted in the configuration of points in a two-dimensional space as shown in Fig. 6.

Dimension 1 represents a monotonic ordering of the stimuli in terms of their statistical properties, where the high negative values represent patterns having high variance and the high positive values represent patterns that have the lowest variance. Patterns are seen to line up along this dimension, then, in terms of their degree of structuredness: low-structured or random patterns toward the negative end of the continuum and the highly structured patterns at the positive end.

The two-dimensional configuration suggests that, as the patterns become less structured in terms of their variance, stimuli representing the different local properties tended to be further away from one another in the space. From this solution, one could conclude that unpracticed Ss did order textured displays of different local properties in terms of their statistical distributions, but as the variance of the distribution increased, a pair of patterns representing different local properties, but having similar statistical properties, appeared to be further apart in the perceptual space. As expected, the traditional scaling model resulted in a similar perceptual space for this same set of experimental data. A two-dimensional traditional solution for the

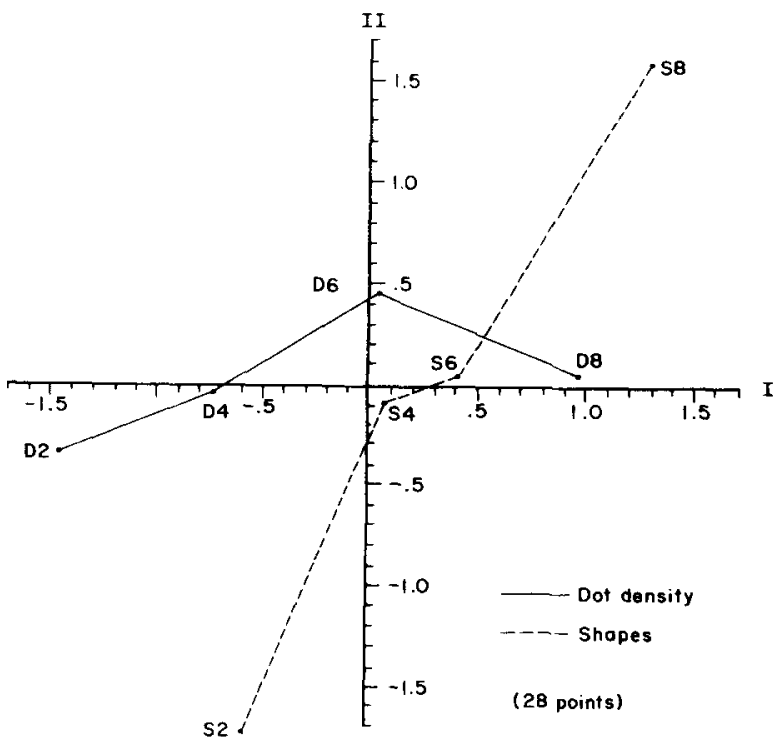

Fig. 7. Traditional solution dimensions: no-practice group. 
unpracticed Ss showed the same ordering of points, as a function of variance, as was found with the nonmetric method.

\section{DISCUSSION}

Several solutions were attempted for the practiced Ss and they were difficult to interpret. Even if only five different Ss' data with five different trials were used to arrive at some reasonable sample size for achieving a solution with eight stimuli, the space was not at all similar to that shown by the unpracticed Ss. The data suggest that these practiced $S s$ had difficulty scaling the stimuli, because of earlier participation in the detection study. That is, during the detection study, they were required to respond either same or different to a pair of stimuli differing only in their statistical distributions, not local property. The most obvious difference from prior practice was a local property difference, rather than differences in the statistics of the patterns, all of which served to make the transition from the detection to the scaling study a most difficult one. The experiments suggested that when Ss had prior practice with detection of the textured stimuli, they were not able to scale these stimuli in a perceptual space similar to that of the nonpracticed group of Ss. This suggests a question for further research. What would the detection data look like for a group of Ss who had prior practice in scaling differences in the statistical distributions of textured displays?

In general, however, the "unpracticed" Ss ordered the displays in terms of their statistical properties for the different local properties of dot density and shapes.

\section{REFERENCES}

DIEDERICH, G., MESSICK, S., \& TUCKER, L. A general least squares solution for successive intervals. Psychometrika, 1957, 22, 159-173.

GIBSON, J. J. Perception of the visual world. Boston: Houghton Mifflin, 1950.

KRUSKAL, J. B. Multidimensional scaling by optimizing goodness of fit to a nonmetric hypothesis. Psychometrika, 1964a, 29, 1-27.

KRUSKAL, J. B. Nonmetric multidimensional scaling: A numerical method. Psychometrika, 1964b, 29, 115-129.

PICKETT, R. M. The perception of random visual texture. In W. W. Dunn (Ed.), Models for the perception of speech and visual form. Cambridge, Mass.: M.I.T. Press, 1967.

PICKETT, R. M. Perceiving visual texture: A literature survey. Technical Report 68-12. Aerospace Medical Research Laboratories, WrightPatterson Air Force Base, Ohio, 1968.

ROSENFELD, A. On models for the perception of visual texture. In W. W. Dunn (Ed.), Models for the perception of speech and visual form. Cambridge, Mass.: M.I.T. Press, 1967.

SHEPARD, R. The analysis of proximities: Multidimensional scaling with an unknown distance function. I. Psychometrika, 1962, 27, 125-140.

SHEPARD, R. The analysis of proximities: Multidimensional scaling with an unknown distance function. II. Psychometrika, 1962, 27, 219-246.

\section{NOTES}

1. This research was performed in partial fulfillment of the requirements of the degree of Doctor of Philosophy at the University of Maryland. The work was supported in part by Grant NsG 398 from the National Aeronautics and Space Administration to the University of Maryland. The author gratefully acknowledges the invaluable help of Nancy S. Anderson and Azriel Rosenfeld. Portions of this paper were presented at the 1967 annual meeting of the Psychonomic Society, Chicago, Illinois.

2. Address: Center for Naval Analyses, 1401 Wilson Blvd., Arlington, Virginia 22209.

(Accepted for publication April 14, 1969.) 